Josef BukaC (Jaromer-Josefov)

\title{
POLYNOMIALS ASSOCIATED WITH EXPONENTIAL REGRESSION
}

Abstract. Fitting exponentials $a+b e^{c x}$ to data by the least squares method is discussed. It is shown how the polynomials associated with this problem can be factored. The closure of the set of this type of functions defined on a finite domain is characterized and an existence theorem derived.

1. Introduction. Suppose we are given integers $X_{i}$ and real numbers $Y_{i}$ for $i=1, \ldots, t$. It is convenient if $Y_{i}$ are integers but not necessary. The $X_{i}$ and $Y_{i}$ are not random variables, we are dealing with a numerical assignment. Instead of using $a+b \exp (c x)$ we prefer the model $a+b R^{x}$ and ask for $a, b$, and $R>0$ that would minimize $\sum\left(a+b R^{X_{i}}-Y_{i}\right)^{2}$. If there is such a minimum, then the partial derivatives with respect to $a, b, R$ are equal to zero at a point at which the minimum is located and, if $b \neq 0$, we are led to the equations

$$
\begin{gathered}
a t+b \sum R^{X_{i}}-\sum Y_{i}=0, \\
a \sum R^{X_{i}}+b \sum R^{2 X_{i}}-\sum Y_{i} R^{X_{i}}=0, \\
a \sum X_{i} R^{X_{i}}+b \sum X_{i} R^{2 X_{i}}-\sum Y_{i} X_{i} R^{X_{i}}=0 .
\end{gathered}
$$

If there exists a solution to this system of equations for some $R$, then $a, b,-1$ are the coefficients of a linear combination of the columns of the matrix

$$
\left[\begin{array}{ccc}
t & \sum R^{X_{i}} & \sum Y_{i} \\
\sum R^{X_{i}} & \sum R^{2 X_{i}} & \sum Y_{i} R^{X_{i}} \\
\sum X_{i} R^{X_{i}} & \sum X_{i} R^{2 X_{i}} & \sum Y_{i} X_{i} R^{X_{i}}
\end{array}\right]
$$

yielding the zero vector.

2000 Mathematics Subject Classification: 62J02, 65D10, 65D15.

Key words and phrases: nonlinear regression; existence; closure. 
That can only happen if the column vectors are linearly dependent, for which a necessary and sufficient condition is that the determinant of the matrix is zero for a given $R$. If the values of $X_{i}$ are nonnegative integers, the determinant may be written as a polynomial function of one variable. The positive roots of the polynomial give the values for which the sum of squares may be minimal.

We know that a polynomial does not have more roots than its degree. In our case it is three times the maximum of $X_{i}$. If $X_{i}$ take on values 1 through 5 , the determinant gives a polynomial of degree fifteen. It is generally known that finding roots of polynomials of high degrees is numerically very difficult.

This method and other details may be found in Gregg et al. (1964) (see also the survey paper Pimentel-Gomes (1953)). The origin of the idea is attributed to Pimentel-Gomes and Malavolta (1949).

2. Basic properties. Let $X=\left(X_{1}, \ldots, X_{t}\right)^{\prime}$ and $Y=\left(Y_{1}, \ldots, Y_{t}\right)^{\prime}$ be vectors for which we define the determinant $D(R, X, Y)$ as above. We can see immediately that

$$
\begin{aligned}
& D(R, X, Y)=-D(1 / R,-X, Y) \\
& D(R, X, Y)=-D(R, X,-Y) \\
& D(R, X, Y)=D(1 / R,-X,-Y)
\end{aligned}
$$

Let $E$ be the vector consisting of ones and $u$ a positive constant. We also have

$$
\begin{aligned}
D(R, u E+X, Y) & =R^{u} D(R, X, Y), \\
D(R, X, u E+Y) & =D(R, X, Y), \\
D(R, X, u Y) & =u D(R, X, Y), \\
D(R, u X, Y) & =u D\left(R^{u}, X, Y\right) .
\end{aligned}
$$

We can use these facts to modify $X$ to get a smaller degree of the polynomial. We can subtract, for example, the minimal value of $X$ from all its entries or we can divide all the entries by their greatest common divisor. We can also change the vector $Y$ to keep the coefficients of the polynomial as small as possible.

We conclude that if $Z^{\prime}=u E^{\prime}+v Y^{\prime}$, where $u, v$ are constants and $E^{\prime}=$ $(1, \ldots, 1)$, then the polynomials obtained from the determinants $D(R, X, Z)$ $=v D(R, X, Y)$ differ only by a constant factor $v$. This is obvious but it is less obvious that the converse is also true. If the polynomials $D(R, X, Z)$ and $D(R, X, Y)$ differ only by a constant factor, then $Z^{\prime}=u E^{\prime}+v Y^{\prime}$ for some $u$ and $v$. The proof of this fact is tedious and too long to be presented here. 
3. Factoring $D(R, X, Y)$ as a polynomial in $R$. The fact that $(R-1)^{3}$ divides $D(R, X, Y)$ when the components of $X$ are equidistant, is mentioned in Pimentel-Gomes (1953) and is attributed to Nogueira (1950). It is straightforward but tedious to extend it to the general case.

If we let $R=1$, we see that the value of the determinant is zero. Even though the value $R=1$ does not have any suitable interpretation, this root enables us to factor the polynomial. We can try to investigate the determinant as a function of $R$ and calculate its derivative at $R=1$. As a reward we get a surprise, this derivative is zero no matter what $X$ and $Y$ look like. We even get a bigger surprise when we find out that the second derivative is always zero at $R=1$ regardless of $X$ and $Y$. It took a while to calculate the third derivative at $R=1$; it is, after skipping the obvious subscripts,

$$
\begin{aligned}
& 3 \sum X\left(\sum X^{2} \sum Y X+\sum X^{3} \sum Y-\sum X \sum Y X^{2}\right) \\
& +3 t\left(\sum X^{2} \sum Y X^{2}-\sum X^{3} \sum Y \sum X\right)-3 \sum X^{2} \sum X^{2} Y .
\end{aligned}
$$

The Taylor expansion centered at $R=1$ of the polynomial $P(R)$ of some degree $M$ is

$$
\begin{aligned}
P(R)= & P(1)+P^{\prime}(1)(R-1)+P^{\prime \prime}(1)(R-1)^{2} / 2 \\
& +P^{\prime \prime \prime}(1)(R-1)^{3} / 6+\ldots+P^{(M)}(1)(R-1)^{M} / M ! .
\end{aligned}
$$

Since $P(1)=P^{\prime}(1)=P^{\prime \prime}(1)=0,(R-1)^{3}$ is a factor of $P(R)$. It is not only the decrease in the degree of the polynomial that we achieve, we also discard multiple roots which can cause trouble in numerical calculations.

4. Examples. We give three examples to illustrate various situations.

\begin{tabular}{cccccc}
\hline \multirow{2}{*}{$\begin{array}{c}\text { Example } \\
\text { 1 }\end{array}$} & \multicolumn{2}{c}{ Example } & \multicolumn{2}{c}{ Example } \\
\hline$y$ & $x$ & $y$ & $x$ & $y$ & $x$ \\
0 & 0 & 1 & 0 & 0 & 0 \\
0 & 1 & 2 & 1 & 5 & 1 \\
0 & 2 & 3 & 2 & 2 & 2 \\
1 & 3 & 1 & 3 & 7 & 3 \\
\hline
\end{tabular}

Only four observations were considered in each example and data made up to suit the given purpose. We cannot present data with a larger number of observations because the degrees of polynomials would be prohibitive.

The purpose of these examples is to show that difficulties a routine use of statistical software may cause are for real. It is obvious that the usual numerical methods of finding minima will fail if the minima are not there. 
Example 1 . We get the polynomial $2 R^{3}+3 R^{2}+4 R+1$. Positive coefficients mean no positive root and no $a, b, R>0$ at which a local minimum would be located. The infimum of the sum of squares is zero since we can pick $R=N, a=0, b=1 / N^{3}$, where $N$ is a positive integer, and the sum of squares is

$\left(0-1 / N^{3}\right)^{2}+\left(0-N / N^{3}\right)^{2}+\left(0-N^{2} / N^{3}\right)^{2}+\left(1-N^{3} / N^{3}\right)^{2}<3 / N^{2}$.

For every $\varepsilon$ there is an $R$ such that the sum of squares is less than $\varepsilon$, thus its infimum is zero. For no positive value of $R$ can this infimum be attained, because $a+b R^{x}$ is strictly monotone for $R>0$, disregarding $R=1$ and $b=0$.

EXAmple 2. This example yields the polynomial $2 R^{3}+7 R^{2}+6 R+5$. When we try an iterative method, it is a gross error to believe that the parameters converge to a minimal solution. After repeated starts we can see that they diverge even though the sum of squares is stable. It is interesting to try to fit a straight line to this set of data by the least squares method. The sum of squares is equal to 4.2. No matter how hard we try, we never get a smaller sum of squares using $a+b R^{x}$, even the best guesses gave a little bit more than 4.2 .

EXAmple 3. The polynomial we get is

$$
P(R)=R^{3}-3 R^{2}-3 R+1=(R+1)\left(R^{2}-4 R+1\right),
$$

the equation $R^{2}-4 R+1=0$ has $R_{1}=2+\sqrt{3}$ and $R_{2}=2-\sqrt{3}$ as its roots. It can be shown that there are no local minima at $R_{1}$ or $R_{2}$. It can also be seen that local minima are obtained in the closure of but outside the set of functions $a+b R^{x}$.

5. Closure. We have dealt with some examples first to make it clear that the set of functions $a+b R^{x}$ is not closed in the sense that a convergent sequence of functions from this set need not have a limit in this set.

Let us consider the closure of the set of functions $a+b R^{x}$ defined on a finite set. If there is, in this closure, another function with the sum of squares smaller than the sum of squares for any of the functions $a+b R^{x}$, then there is no minimal solution among the functions $a+b R^{x}$. It is conceivable that there might even be some local minima with the sum of squares greater than the infimum but the infimum could be attained by a function in the closure different from $a+b R^{x}$. It would be nice to know what kind of functions form this closure. We could then find the function with the minimal sum of squares in the closure and the question would be resolved.

6. Characterization of the closure. Let us assume that we have $t$ points $0=x_{1}<\ldots<x_{t}$ and let us consider the set of all functions defined 
at these points. All these points and function values do not have to be integers any more. It is essential that, without loss of generality, we may indeed assume $x_{1}=0$ and $F\left(x_{1}\right)=0$, since we can always add a constant or use a substitution to translate the original problem into one with $x_{1}=0$ and $F\left(x_{1}\right)=0$ and backwards.

We take a Euclidean metric for the values of the functions as a distance $d(F, G)=\left(\sum\left(F\left(x_{i}\right)-G\left(x_{i}\right)\right)^{2}\right)^{1 / 2}$ where $F$ and $G$ are two functions defined at $x_{i}$ for $i=1, \ldots, t$. The distance $d(F, G)$ corresponds to the sum of squares of differences in an obvious manner.

By definition, a function $F$ is in the closure of a set of functions if there is a sequence of functions in this set converging to $F$ with respect to the distance $d(F, G)$. Since the number of points $0=x_{1}<\ldots<x_{t}$ is finite, it is easy to see that the convergence of a sequence of functions with respect to the distance $d(F, G)$ is equivalent to the pointwise convergence. This fact is important because the pointwise convergence is easier to work with.

Assume that $F$ is in the closure of functions $a+b R^{x}$ defined on $0=x_{1}<$ $\ldots<x_{t}$. Then there is a sequence $a_{N}+b_{N} R_{N}^{x_{i}}$ converging to $F\left(x_{i}\right)$ for every $i=1, \ldots, t$. The aim is to show what properties $F$ has.

We may start with a special but illustrative case first. The case of $b_{N}=0$ may be treated by discarding $a_{N}+b_{N} R_{N}^{x}$ with $b_{N}=0$ if there are finitely many members with $b_{N}=0$. In the case of an infinite subsequence with $b_{K}=0$, it does not matter what $R_{K}$ is; if $b_{K}=0$ then $a_{K}+b_{K} R_{K}^{x}=a_{K}$. Since $F\left(x_{1}\right)=0$, we get $\lim a_{K}=0$ and, as a result, $F\left(x_{i}\right)=\lim \left(a_{K}+\right.$ $\left.b_{K} R_{K}^{x_{i}}\right)=\lim a_{K}=0$ for all $i$, that is, $F(x)$ must be a constant function. It obviously does not matter what the rest of the sequence $a_{N}+b_{N} R_{N}^{x}$ may look like, it converges and it does so to $F(x)$. This is the basic idea that the proofs are based on.

If we now assume that $b_{N} \neq 0$ for all $N$, the proof will depend on the assumptions about $R_{N}$ and, accordingly, the classification of the limits of $a_{N}+b_{N} R_{N}^{x}$ will be made. Our basic assumption is that $R_{N}>0$ for all $N$, no assumptions are made at this point regarding $a_{N}$ or $b_{N} \neq 0$ and the questions of convergence of $a_{N}$ or $b_{N}$ are studied only when they are needed to determine the convergence of $a_{N}+b_{N} R_{N}^{x}$ as functions.

After the case that $R_{N}$ is unbounded we get the fact that $R_{N}$ is bounded and, therefore, we can pick a subsequence $R_{K}$ that is convergent. Various possibilities of limits of this subsequence are then discussed.

7. The case of $R_{N}$ unbounded. If the sequence $R_{N}$ is not bounded, there must be a subsequence $a_{K}+b_{K} R_{K}^{x}$, convergent to $F(x)$ at $x_{1}, \ldots, x_{t}$, for which $R_{K}$ goes to infinity. We could even pick $R_{K}$ increasing. At $x_{1}=0$, the value of $a_{K}+b_{K} R_{K}^{x}$ is equal to $a_{K}+b_{K}$ for any $R$. If $a_{K}+b_{K} R_{K}^{0}$ converges to $F\left(x_{1}\right)=0, \lim \left(a_{K}+b_{K}\right)=0$ must hold. Since $R_{K}$ converges 
to infinity and $x_{t}>0, b_{K}$ must go to zero, otherwise there would be $\varepsilon>0$ such that $\left|b_{K}\right|>\varepsilon$ for infinitely many $K,\left|b_{K}\right|\left(R_{K}^{x_{t}}-1\right)$ would not be bounded and $a_{K}+b_{K} R_{K}^{x_{t}}=a_{K}+b_{K}+b_{K} R_{K}^{x_{t}}-b_{K}=\left(a_{K}+b_{K}\right)+b_{K}\left(R_{K}^{x_{t}}-1\right)$ would not be bounded, thus $\lim b_{K}=0$ must hold and we get $\lim a_{K}=0$.

Now we can see that $\lim b_{K} R_{K}^{x_{t}}=F\left(x_{t}\right)$ and $\lim b_{K} R_{K}^{x_{i}}$ is, for $i<t$, equal to $\lim b_{K} R_{K}^{x_{t}} /\left(R_{K}^{x_{t}-x_{i}}\right)$. Since the numerator has a finite limit $F\left(x_{t}\right)$ and the denominator goes to infinity, the limit of the fraction is zero.

8. Subsequence $R_{K}$ going to zero. Let there exist a subsequence $R_{K}$ converging to zero. As before, it is clear that $\lim \left(a_{K}+b_{K}\right)=F(0)=0$. It follows from the boundedness of $a_{K}+b_{K} R_{K}^{x}$ that there is a $B_{1}$ such that

$$
\left|a_{K}+b_{K}+b_{K} R_{K}^{x}-b_{K}\right|=\left|a_{K}+b_{K} R_{K}^{x}\right|<B_{1}
$$

for all $K$ and from $\lim \left(a_{K}+b_{K}\right)=0$ it follows that there is a $B_{2}$ for which $\left|b_{K}\left(R_{K}^{x}-1\right)\right|<B_{2}$. Therefore $\left|b_{K}\right|<B_{2} /\left(1-R_{K}^{x}\right)$ starting from some $K$, thus $b_{K}$ is a bounded sequence, because $\lim R_{K}^{x}=0$. Thus $b_{K} R_{K}^{x}$ converges to zero for $x>0$ and we must have $\lim a_{K}=F\left(x_{2}\right)=\ldots=F\left(x_{t}\right)$.

9. Subsequence with a positive limit other than one. Let there be a subsequence $R_{K}$ with $\lim R_{K}=R$, where $R$ is positive, finite, and different from one. We again have $\lim \left(a_{K}+b_{K}\right)=0$ and we know that $\lim \left(a_{K}+b_{K} R_{K}^{x_{t}}\right)=F\left(x_{t}\right)$. Since $a_{K}+b_{K} R_{K}^{x_{t}}=a_{K}+b_{K}+b_{K} R_{K}^{x_{t}}-b_{K}=$ $a_{K}+b_{K}+b_{K}\left(R_{K}^{x_{t}}-1\right)$ converges to $F\left(x_{t}\right)$, we have $\lim b_{K}\left(R_{K}^{x_{t}}-1\right)=F\left(x_{t}\right)$. We know, because of continuity, that $\lim \left(R_{K}^{x_{t}}-1\right)=R^{x_{t}}-1$, and the following will also hold:

$$
\lim b_{K}=\lim b_{K}\left(R_{K}^{x_{t}}-1\right) /\left(R_{K}^{x_{t}}-1\right)=F\left(x_{t}\right) /\left(R^{x_{t}}-1\right) .
$$

Set $b=\lim b_{K}$. Then we can see that $a=\lim a_{K}=-b$. Thus $a_{K}+b_{K} R_{K}^{x}$ converges to $a+b R^{x}$.

10. Subsequence with limit one. (a) If there is a subsequence $R_{K}$ with all elements one, then $a_{K}+b_{K} R_{K}^{x}=a_{K}+b_{K}$. We know that the limit of this sequence is zero, which implies that $F\left(x_{i}\right)=0$ for all $i$.

(b) Let each $R_{K}$ be different from one. Set $U_{K}(x)=a_{K}+b_{K} R_{K}^{x}$. Then $U_{K}(0)=a_{K}+b_{K}$. Define $D_{K}(x)=U_{K}(x)-U_{K}(0)$. Then we get $D_{K}(x)=$ $b_{K}\left(R_{K}^{x}-1\right)$. This difference will be treated separately and its limit will be added to $\lim \left(a_{K}+b_{K}\right)=0$. We will need the following limit for $R$ going to one while $x$ and $x_{t}$ are fixed:

$$
\lim _{R \rightarrow 1}\left(R^{x}-1\right) /\left(R^{x_{t}}-1\right)=x / x_{t} .
$$

Since $a_{K}+b_{K} R_{K}^{x_{t}}$ converges to $F\left(x_{t}\right)$ or $\lim U_{K}\left(x_{t}\right)=F\left(x_{t}\right)$ and at the same time $\lim U_{K}(0)=F\left(x_{1}\right)=0$, we have $\lim D_{K}\left(x_{t}\right)=F\left(x_{t}\right)$. Thus

$$
\lim D_{K}\left(x_{t}\right)=\lim b_{K}\left(R_{K}^{x_{t}}-1\right)=F\left(x_{t}\right) .
$$


We can use the limit we found beforehand to get

$$
\lim D_{K}(x)=\lim b_{K}\left(R_{K}^{x}-1\right)=\lim b_{K}\left(R_{K}^{x_{t}}-1\right) \frac{R_{K}^{x}-1}{R_{K}^{x_{t}}-1} .
$$

That gives $\lim D_{K}(x)=x F\left(x_{t}\right) / x_{t}$ and shows that the limit of $D_{K}(x)$ exists for $x>0$ and is proportional to $x$.

As the limit of $a_{K}+b_{K} R_{K}^{x}$ is zero for $x_{1}=0$ and the value of the function $F$ is the sum of its value at zero and the difference, we conclude that $F$ is a linear function $m x$, where $m=F\left(x_{t}\right) / x_{t}$.

11. Completion of characterization. Now we can work our way back and see that the closure consists of functions we get by adding a constant or by linear substitution. As a result, we can describe the functions in the closure in the following manner.

THEOREM. If there are $t \geq 3$ points $x_{1}<\ldots<x_{t}$, then the closure of the set of functions $a+b R^{x}$, where $0<R \neq 1$, defined at these points is formed by the following functions:

(1) the functions constant except at the first point $x_{1}$, that is, $F\left(x_{1}\right)$ is arbitrary, $F\left(x_{2}\right)=F\left(x_{3}\right)=\ldots=F\left(x_{t}\right)$;

(2) the functions constant except the last point $x_{t}$, that is, $F\left(x_{1}\right)=$ $F\left(x_{2}\right)=\ldots=F\left(x_{t-1}\right)$, arbitrary $F\left(x_{t}\right)$;

(3) $a+m x$;

(4) $a+b R^{x}$

Proof. What is left to prove is that all the functions of the four types are in the closure. Let $F\left(x_{i}\right)=0$ for $i<t$ and $F\left(x_{t}\right)=1$. As in Example 1 , let $a=0, b=1 / N^{x_{t}}$, and $R=N, N$ positive integer. We get the sum of squares

$$
\begin{aligned}
\left(0-N^{x_{1}} / N^{x_{t}}\right)^{2}+\ldots+\left(0-N^{x_{t-1}} / N^{x_{t}}\right)^{2}+ & \left(1-N^{x_{t}} / N^{x_{t}}\right)^{2} \\
& <(t-1) / N^{2\left(x_{t}-x_{t-1}\right)} .
\end{aligned}
$$

As $x_{t}-x_{t-1}>0$, by choosing $N$ sufficiently large the sum of squares can be made arbitrarily small. The general case can be obtained by choosing suitable $a$ and $b$.

When $F\left(x_{1}\right)=1$ and $F\left(x_{i}\right)=0$ for $i>1$, we proceed in the similar manner. Let $a=0, b=1 / N^{x_{1}}$, and $R=1 / N, N$ natural, and write down the sum of squares of deviations.

Let $F(x)=q+m x, m \neq 0$. Choose any sequence $R_{N}$ convergent to one with all $R_{N}$ different from one. The sequences $a_{N}$ and $b_{N}$ can be obtained from the equations

$$
\begin{aligned}
& a_{N}+b_{N} R_{N}^{0}=q, \\
& a_{N}+b_{N} R_{N}^{x_{t}}=q+m x_{t} .
\end{aligned}
$$

The determinant of the system is $R_{N}^{x_{t}}-1 \neq 0$ and we obtain the sequence 
$a_{N}+b_{N} R_{N}^{x}$. Define $D_{N}(x)=U_{N}(x)-U_{N}(0)=b_{N}\left(R_{N}^{x}-1\right)$ and compute $\lim D_{N}(x)=\lim b_{N}\left(R_{N}^{x_{t}}-1\right) \frac{R_{N}^{x}-1}{R_{N}^{x_{t}}-1}=m x_{t}\left(x / x_{t}\right)=m x$,

because $b_{N} R_{N}^{x_{t}}-b_{N}=a_{N}+b_{N} R_{N}^{x_{t}}-\left(a_{N}+b_{N} R_{N}^{0}\right)=q+m x_{t}-q=m x_{t}$ by definition of $a_{N}$ and $b_{N}$.

12. Existence of a minimal solution in the closure. The closure of the set of functions $a+b R^{x}$ has been described and what is left is the proof of the existence of a minimal solution in this closure.

TheOREM. Let $\left(y_{i}, x_{i}\right)$ for $i=1, \ldots, t$ be pairs of real numbers with at least three $x$ 's distinct. Among the functions $F(x)$ in the closure of functions of the type $a+b R^{x}$ defined at $x_{1}, x_{2}, \ldots, x_{t}$ there exists a function $F_{0}(x)$ that minimizes the sum of squares $\sum_{i=1}^{t}\left(F\left(x_{i}\right)-y_{i}\right)^{2}$.

Proof. We consider $\left(y_{i}, x_{i}\right)$ for $i=1, \ldots, t$. We may have $x_{i}=x_{j}$ for some $i \neq j$, which is what statisticians call repeated observations. We can choose one fixed function $a_{0}+b_{0} R_{0}^{x}$ and determine the sum of the squares of the deviations from $y_{i}$. By a closed ball $B_{0}$ in the set of functions defined on the $x_{i}$ for $i=1, \ldots, t$ we will mean the subset of those functions for which $\sum\left(F\left(x_{i}\right)-y_{i}\right)^{2} \leq \sum\left(a_{0}+b_{0} R_{0}^{x_{i}}-y_{i}\right)^{2}$. We are not dealing exactly with a closed ball as usually defined in topology because we have to consider repeated observations. It is clear that this ball $B_{0}$ is nonempty, closed and bounded, because the sum of squares is a continuous function and the image under this mapping is a closed interval. One of its endpoints is $\sum\left(a_{0}+\right.$ $\left.b_{0} R_{0}^{x_{i}}-y_{i}\right)^{2}$; the other endpoint is the sum of the squares of the deviations $\sum\left(F_{\min }\left(x_{i}\right)-y_{i}\right)^{2}$ of the function $F_{\min }$, the values of which are arithmetical averages of $y_{i}$ calculated for each value $x_{i}$, for these averages minimize the sum of squares of deviations at each value of $x_{i}$ separately.

If there are repeated values of $x_{i}$, we may not have exactly a ball. This is especially the case when for some $i \neq j$ the values of $y_{i}$ and $y_{j}$ may be different, whereas $x_{i}$ and $x_{j}$ are the same. On the other hand, if, for some $i, x_{i}$ is different from all other $x_{j}, j \neq i$, we have $F_{\min }\left(x_{i}\right)=y_{i}$.

To show that the sums of squares of deviations take on all the values in the interval we set $F_{C}\left(x_{i}\right)=F_{\min }\left(x_{i}\right)$ for $i>1$ while $F_{C}\left(x_{1}\right)=F_{\min }\left(x_{1}\right)+C$ for $i=1$. Thus, by picking the right $C \geq 0$, we get any desired sum of squares of deviations from $\sum\left(F_{\min }\left(x_{i}\right)-y_{i}\right)^{2}$ to infinity.

The intersection of the closed ball $B_{0}$ with the closure of the set of functions $a+b R^{x}$ is nonempty, closed and bounded, thus it is a nonempty and compact set and we know that a continuous mapping, such as the sum of squares of deviations, as a mapping of $F$, takes on its infimum on a set like that at some point $F_{0}$. 
13. Applications and comments. Another model may be given by the function $b \exp (c x)=b R^{x}$ defined on a finite set. To find out how the closure of the set of functions of this type looks like, we would have to discuss three cases: $R_{K}$ unbounded, $R_{K}$ going to zero, and $R_{K}$ going to some finite positive limit. $R=1$ is acceptable for it defines a constant function.

Other frequently used models derived from $a+b R^{x}$ are the logistic function and the Gompertz function, the former being just the reciprocal of $a+b R^{x}$, the latter the exponential function of $a+b R^{x}$. It is clear that in both cases the closure can be described in a similar manner. It is just that one has to be careful about the division by zero.

Since it is hard to find all the positive real roots of polynomials of large degrees, users of statistical software will use iterative methods to minimize the sum of squares. But we now know that the result has to be compared against minimal sums of squares of other types of functions in the closure of the set of exponential ones.

To determine if the reciprocal of a linear function yields a better sum of squares than the logistic one we have to deal with two nonlinear regression problems. The graphical visualization of the reciprocal of the logistic function is of great value to see how close it is to the straight line.

\section{References}

J. V. Gregg, C. H. Hossell and J. T. Richardson (1964), Mathematical Trend Curves: an Aid to Forecasting, Oliver and Boyd, Edinburgh.

I. R. Nogueira (1950), Sôbre una propriedade da equação utilizada para a interpolação da lei de Mitscherlich, Anais Esc. Sup. Agr. Luiz de Queiroz 7, 105-108.

F. Pimentel-Gomes (1953), The use of Mitscherlich's regression law in the analysis of experiments with fertilizers, Biometrics 9, 498-516.

F. Pimentel-Gomes and E. Malavolta (1949), Aspectos matematicos e estatisticos da lei de Mitscherlich, Anais Esc. Sup. Agr. Luiz de Queiroz 6, 193-229.

Bulharska 298

55102 Jaromer-Josefov

Czech Republic 